\title{
A phylogenetically conserved APETALA2/ETHYLENE RESPONSE FACTOR, ERF12, regulates Arabidopsis floral development
}

\author{
J. W. Chandler ${ }^{1}$ iD $\cdot$ W. Werr ${ }^{1}$
}

Received: 5 March 2019 / Accepted: 30 October 2019 / Published online: 5 December 2019

(c) The Author(s) 2019

\begin{abstract}
Key message Arabidopsis ETHYLENE RESPONSE FACTOR12 (ERF12), the rice MULTIFLORET SPIKELET1 orthologue pleiotropically affects meristem identity, floral phyllotaxy and organ initiation and is conserved among angiosperms.

Abstract Reproductive development necessitates the coordinated regulation of meristem identity and maturation and lateral organ initiation via positive and negative regulators and network integrators. We have identified ETHYLENE RESPONSE FACTOR12 (ERF12) as the Arabidopsis orthologue of MULTIFLORET SPIKELET1 (MFS1) in rice. Loss of ERF12 function pleiotropically affects reproductive development, including defective floral phyllotaxy and increased floral organ merosity, especially supernumerary sepals, at incomplete penetrance in the first-formed flowers. Wildtype floral organ number in early formed flowers is labile, demonstrating that floral meristem maturation involves the stabilisation of positional information for organogenesis, as well as appropriate identity. A subset of erf 12 phenotypes partly defines a narrow developmental time window, suggesting that $E R F 12$ functions heterochronically to fine-tune stochastic variation in wild type floral number and similar to MFS1, promotes meristem identity. ERF12 expression encircles incipient floral primordia in the inflorescence meristem periphery and is strong throughout the floral meristem and intersepal regions. ERF12 is a putative transcriptional repressor and genetically opposes the function of its relatives DORNRÖSCHEN, DORNRÖSCHEN-LIKE and PUCHI and converges with the APETALA2 pathway. Phylogenetic analysis suggests that ERF12 is conserved among all eudicots and appeared in angiosperm evolution concomitant with the generation of floral diversity.
\end{abstract}

Keywords Angiosperm evolution $\cdot$ APETALA2 $\cdot$ ETHYLENE RESPONSE FACTOR $12 \cdot$ Floral transition $\cdot$ Meristem identity $\cdot$ MULTIFLORET SPIKELET $1 \cdot$ Phyllotaxy $\cdot$ Supernumerary sepals

\section{Introduction}

Plant reproductive development involves successive phase changes, whereby vegetative apical meristems that initiate leaf primordia at their periphery become inflorescence meristems (IMs) and initiate floral meristems, which in turn,

Electronic supplementary material The online version of this article (https://doi.org/10.1007/s11103-019-00936-5) contains supplementary material, which is available to authorized users.

J. W. Chandler

john.chandler@uni-koeln.de

W. Werr

werr@uni-koeln.de

1 Developmental Biology, Institute of Zoology, Cologne Biocenter, University of Cologne, Zuelpicher Straße 47b, 50674 Cologne, Germany generate floral organs. The gene regulatory networks that regulate these meristem identities have been elucidated in detail (Ó’Maoiléidigh et al. 2014), together with those involved in the floral transition in response to seasonal cues, for representative dicot and monocot species (Shrestha et al. 2014). Reproductive phase change also depends on endogenous age-related competencies that integrate into these genetic networks via miRNAs (Teotia and Tang 2015). Flower development thus proceeds from the complex coordinated specification of meristem identity, and floral organ initiation and identity, via positive and negative regulators and network integrators.

Instead of leaf initiation at the shoot apical meristem switching to flower production within a single plastochron, the acquisition and maintenance of floral meristem (FM) identity are not robust and can revert to IM identity in several species in response to environmental conditions 
(McCullough et al. 2010; Tooke et al. 2005). Furthermore, floral organs do not initiate simultaneously within the FM in concentric whorls: instead, outer whorl organs initiate sequentially along an abaxial/adaxial axis, with a stereotypy that can be disrupted by bract founder cell recruitment, or gene mutations that lead to pentameric asymmetry (Chandler and Werr 2014). The floral transition and floral organ initiation therefore represent the outread of multiple components and incremental signals and consist of several distinct phases.

Threshold expression models have been invoked to explain the function of some master regulators of the floral transition, such as $L E A F Y(L F Y)$ (Blázquez et al. 1997), and the control of APETALA2 (AP2) by miRNAs (Chen 2004). Many master regulators such as $L F Y$, function pleiotropically in both reproductive timing and floral identity (Schultz and Haughn 1991; Blázquez et al. 1997), and in addition to regulating the identity of the outer two floral organ whorls, APETALA2 (AP2) represses the floral transition (Yant et al. 2010). Arabidopsis AP2 is the founding member of the large AP2 superfamily of transcription factors, which has been subdivided according to the presence of one or two AP2 domains (Nakano et al. 2006). AINTEGUMENTA (ANT) subclass AP2 members contain two AP2 domains and play developmental roles in Arabidopsis (Horstman et al. 2014) and also mediate resistance to salt stress (Meng et al. 2015). Most single AP2-domain proteins belong to the ethylene response element (ERF), also known as ethylene response element binding protein (EREBP) subgroup (Nakano et al. 2006). Although ERF proteins mediate biotic and abiotic stress responses in many taxa (Dey and Corina Vlot 2015: Müller and Munné-Bosch 2015), the group VIIIb members DORNRÖSCHEN (DRN), DORNRÖSCHEN-LIKE (DRNL), LEAFY PETIOLE and PUCHI also regulate development in diverse plant species (Chandler 2018). Given the high sequence conservation of the AP2 domain among AP2 family members, genetic redundancy is a common feature. For example, ANT-like genes provide distinct individual contributions towards flower development, but function redundantly with $A N T$ (Krizek 2015), and DRN, DRNL and $P U C H I$ redundantly regulate organ number and floral meristem identity (Chandler and Werr 2017). The subtle nature of individual mutant phenotypes and the ubiquity of higher orders of genetic redundancy, suggest that the full repertoire of developmental functions of AP2 family members might not yet have been elucidated.

The comparative analysis of AP2 transcription factor functions can inform their evolution and establish plesiomorphic functions. For example, AP2/ERF members in monocots have revealed evolutionary divergence and functional conservation. In rice, the AP2-type genes SUPERNUMERARY BRACT and OSINDETERMINATE SPIKE$L E T 1$ synergistically determine inflorescence architecture and floral meristem identity (Lee and An 2012). Similarly, INDETERMINATE SPIKELET (IDS) and SISTER OF INDETERMINATE SPIKELET1 (SIDI) play multiple roles in regulating maize inflorescence architecture and although they share some gene targets and functions with Arabidopsis AP2, have also adopted novel functions (Chuck et al. 2008). Monocot counterparts of the clade VIIIb ERF subfamily in Arabidopsis have also neofunctionalised or retained ancestral functions: in contrast to a mild mutant phenotype in Arabidopsis, mutations in the PUCHI orthologues BRANCHED SILKLESS in maize (Colombo et al. 1998) and FRIZZY PANICLE in rice cause much more severe phenotypes (Komatsu et al. 2003). Inter-taxa studies are important not least because Arabidopsis inflorescence development is not representative of the whole plant kingdom, due to the different inflorescence structure of monocots versus dicots, including branching, and multiple meristem identities and transitions (Whipple 2017). Furthermore, grasses such as rice represent a specialised and derived lineage of monocots (Kellogg 2001).

Here, we describe the function of ERF12 in Arabidopsis, the orthologue of rice MULTIFLORET SPIKELET1, which regulates floral organ identity and number, and the timing of spikelet initiation (Ren et al. 2013). Loss of ERF12 function affects pleiotropic aspects of floral development in long days, including floral meristem phyllotaxy, organ merosity in the first-formed flowers, notably in sepal number, and delays the floral transition in long and short days. These phenotypes, which are partly restricted to a narrow developmental time window, suggest that $E R F 12$ possesses a heterochronic function, which regulates the timing of FM specification and fine-tunes the inherent wild type stochastic variation in floral organ merosity in first-formed flowers. Phylogenetic analysis of the ERF12 AP2 domain suggests that ERF12 represents a highly conserved angiosperm-specific innovation.

\section{Materials and methods}

\section{Plant material and growth conditions}

The mutant line SAIL_873_D11 (N877578) contains a T-DNA insertion in the open reading frame of the ERF12 gene (At1G28360) after nucleotide 427 from the ATG start. For erf9, the line SALK_043407 contains a T-DNA insertion after nucleotide 314 from the ATG start in the ERF9 (At5G44210) open reading frame. Both erf12 and erf9 alleles were obtained from NASC. Homozygosity was confirmed by genotyping using primers ERF12genoF/ERF12genoR to genomic DNA spanning the open reading frame, or ERF12F/LB3 for the presence of the insertion for $\operatorname{erf12}$, or using primers ERF9F/ERF9R or Lba1/ERF9R for erf9. The mutant lines $d r n-1, d r n l-1$ and puchi and their genotyping 
have been described previously (Chandler et al. 2007; Chandler and Werr 2017). The ap2-7 allele from NASC (N6241) is in Col-0 background. Plants were grown on soil in the greenhouse in long-day conditions ( $16 \mathrm{~h}$ light, $8 \mathrm{~h}$ dark) or in a controlled environment cabinet at $100 \mu \mathrm{M} \mathrm{m}^{-2}$ light intensity under short days ( $8 \mathrm{~h}$ dark, $16 \mathrm{~h}$ light).

\section{Constructs for expression analysis and complementation}

For expression analysis, 2356 bp ERF12 upstream promoter sequence from the stop codon of the upstream gene to the start codon of the ERF12 open reading frame (AT1G28360) was amplified from Arabidopsis genomic DNA extracted from wild-type plants using the NucleoSpin ${ }^{\circledR}$ Plant II kit (Macherey-Nagel) and primers promERF12F and promERF12R. The resulting fragment was cloned into the TOPO ${ }^{\mathrm{TM}} \mathrm{TA}$ Cloning $^{\mathrm{TM}}$ vector (ThermoFisher Scientific). The primers included flanking AscI (5') and XmaI (3') sites, the latter allowing the introduction the coding region of the green fluorescent protein targeted to the endoplasmic reticulum (erGFP) sequence that terminates in the cauliflower mosaic virus 35S 3'UTR/ polyadenylation signal in front of a second $A s c \mathrm{I}$ restriction site (Comelli et al. 2016). The orientation of $\operatorname{erGFP}$ relative to the $E R F 12$ promoter was confirmed by sequencing, to produce pERF12::erGFP. Two genomic regions containing the ERF12 open reading frame were used for complementation analysis. The shorter version ( $g E R F 12 U p ; 3527 \mathrm{bp}$ ) was amplified using primers promERF12F and gERF12UpR, to include the whole $E R F 12$ upstream promoter region from the stop codon of the upstream gene, the ERF12 open reading frame and the 3 ' untranslated region. Both primers included flanking AscI sites. A longer genomic region ( $g$ ERF12UpDown; $6773 \mathrm{bp}$ ) was constructed, consisting of the whole genomic region, between the open reading frames of the up- and downstream genes, including the ERF12-coding region. This construct was made in two steps, by firstly reamplifying $g E R F 12 U p$ using primers promERF12F and gERF12UpRnew, the latter containing an $\mathrm{XmaI}$ site and cloning the product into TOPO ${ }^{\mathrm{TM}}$ TA Cloning ${ }^{\mathrm{TM}}$ vector (ThermoFisher Scientific) and then introducing a PCR fragment amplified using primers gERF12DSF and gERF12DSR at the 3' end of gERF12Up via the flanking $X m a I$ sites and confirming the correct orientation in relation to the $g E R F 12 U p$ fragment, to create gERF12UpDown. The pERF12::erGFP expression or complementation cassettes were transferred into the binary pGPTV-Asc-Bar (erGFP expression construct) or pGPTV-Asc-Kan (complementation constructs) vectors (Überlacker and Werr 1996) via AscI and were introduced into Agrobacterium tumefaciens GV3101V. Mutant erf12 plants (for complementation) or Col-0 wild type ( $p E R F 12:: e r G F P$ ) were transformed via floral dipping (Clough and Bent 1998). For expression analysis, transgenic plants for $p E R F 12:: e r G F P$ were selected with
BASTA and at least eight independent $\mathrm{T}_{2}$ lines were analysed. For complementation, $T_{1}$ seeds were surface-sterilised with bleach (Lindsey et al. 2017). Plants were selected on sterile half-strength Murashige and Skoog medium for resistance to kanamycin $\left(50 \mu \mathrm{gL}^{-1}\right)$ and were genotyped to confirm the presence of the appropriate transgene using pGPTV-specific pGPTVF/pGPTVR and gene-specific primers ERF12Fgeno/ ERF12Rgeno. $T_{2}$ populations of several independent transgenic lines were analysed for complementation.

\section{Confocal imaging}

Imaging of $p E R F 12:: e r G F P$ expression was performed with a Zeiss LSM 700 confocal laser scanning microscope (CLSM). GFP was excited at $488 \mathrm{~nm}$ and emission was analysed between 502 and $525 \mathrm{~nm}$. Photoshop CS2 software (Adobe) was used to process the CLSM images and Imaris software (Bitplane, Zürich, Switzerland) converted Z-stacks into 3D images.

\section{Phenotypic analysis}

Organ numbers were counted for each whorl of the first fiveformed flowers for 100 plants. Statistical analyses of organ numbers were performed using $t$ tests.

\section{Phylogenetic analysis and phylogenetic shadowing}

Full-length protein sequences were obtained from GenBank (http://www.ncbi.nlm.nih.gov/genbank/) or the PLAZA databases (https://bioinformatics.psb.ugent.be/plaza/) using ERF12 or MFS1 sequences as a query. A phylogenetic tree was compiled using the phylogeny.fr platform (Dereeper et al. 2008); protein sequences were aligned using the MUSCLE program and tree construction employed the maximum likelihood method (PhyML programme) and the Bootstrap procedure (100 replicates). The phylogenetic tree was rendered with TreeDyn software and Bootstrap support values are indicated next to the branches. For phylogenetic shadowing, ERF 12 genomic regions of Brassicacea species (Arabidopsis thaliana, Arabidopsis lyrata, Boechera stricta, Camelina sativa, Eutrema salsugineum and Capsella rubella) were obtained from http://phytozome.jgi.doe.gov/pz/portal.html or http:// www.ncbi.nlm.nih.gov/. Sequences were analysed with online mVista tool with LAGAN alignment (http://genome.lbl.gov/ vista/index.shtml). 


\section{Results}

\section{ERF12 and MFS1 represent an invention that postdates basal angiosperms}

A blast search using the MFS1 amino acid sequence revealed Arabidopsis ERF12 to be a putative orthologue. ERF12 belongs to sub-clade VIIIa of AP2/ERF proteins (Nakano et al. 2006), which contains eight members (ERF3, ERF4, ERF7-12). To address the evolutionary sequence conservation between rice MFS1 and Arabidopsis ERF12, a phylogenetic analysis of the closest available full-length-protein sequences to ERF12 and MFS1 was performed from a range of representative species from different extant phylogenetic groups. All members of clade VIIIa and VIIIb were included in the comparison, together with the closest one, or occasionally two sequences from blast searches made with ERF12 and MFS1 against genomes of mosses and liverworts (Marchantia polymorpha, Physcomitrella patens), basal angiosperms (Amborella trichopoda and the mangoliid Cinnamomum kanehirae), gymnosperms (Gingko biloba, Picea abies, Pinus sylvestris), grass monocots (Brachypodium distachyon, Phyllostachys edulis, Setaria italica, Triticum aestivum, Zea mays), non-grass monocots (Ananas comosum, Musa acuminata, Phalaenopsis equestris, Spirodela polyrhiza, Zostera marina), basal eudicots (Nelumbo nucifera, Papaver somniferum), core eudicots (Glycine max, Medicago truncatula, Populus trichocarpa, Solanum lycopersicum and Vitis vinifera). These sequences formed monocot and eudicot ERF12 subclades, but the closest gymnosperm, moss and liverwort ERF12 and MFS1 homologues clustered more closely with Arabidopsis subclades ERF3 and ERF7 (Fig. 1). Furthermore, the closest ERF12/MFS1 homologue in the angiosperm sister taxon Amborella trichopoda aligned most closely with DRNL (Fig. 1), suggesting that ERF12 proteins represent an invention subsequent to the divergence of gymnosperms and basal angiosperms. The AP2 domain is highly conserved between ERF12 and MFS1 and both proteins share a C-terminal EAR-like domain (Fig. 2a). To assess the conservation of ERF12 regulatory sequences, phylogenetic shadowing of the genomic ERF12 locus among several Brassicaceae species revealed that upstream ERF 12 promoter sequences are more highly conserved than downstream regulatory sequences (Fig. 2b).

\section{ERF12 promotes the floral transition in long and short days}

To characterise the function of ERF12, an erf12 mutant was analysed that contained a T-DNA insertion within the ERF12 open reading frame (Fig. 2a). As a proxy for the timing of the floral transition, the number of rosette and cauline leaves were all significantly higher $(0.001>p<0.0001)$ for erf12 than wild type in both long and short days (Fig. 3a, b).

\section{Loss of ERF12 function preferentially affects sepal and stamen number in long days}

Petal and stamen frequency in erf 12 mutants grown in long days showed the same qualitative trend across the first few flowers in the inflorescence as wild type, but differed quantitatively. The most pronounced erf 12 floral phenotype was significantly more sepals in the first two-formed flowers than wild type (Figs. 3c, e-g, 4a) at a penetrance that was highest in first-formed flowers (24\%) and decreased to $10 \%$ in flower 2 and $1 \%$ in flower 3, but ectopic sepals were observed up to flower 6 . Sepals were also occasionally fused (in $11 \%$ of first-formed flowers; Fig. 3d, e). Mean petal number in the first few erf12 flowers was slightly but not significantly greater than in wild type (Fig. 4b), but more erf12 flowers had either three or five petals than wild type (Fig. 3h, 1; data not shown), which usually had four (Fig. 3d). erf 12 flowers had significantly more stamens than wild type (up to nine) in the first three flowers (Figs. 3j, 4c), preferentially due to significantly more lateral stamens (Fig. 4d); the number of medial stamens in flowers 1 to 5 did not differ between wild type and erf 12 plants (Fig. 4e), but reached seven in both genotypes. Three fused carpels were observed among the first five erf12 flowers at a very low frequency (Fig. 3k; $1 / 200$ plants). Thus, early erf 12 flowers possessed supernumerary organs in all four floral whorls.

Notably, floral organ number in earliest-formed wild-type flowers was non-stereotypic in long-day conditions and was not robust across the inflorescence. No significant variation in sepal number was observed in the earliest-formed flowers (Fig. 4a), but first-formed flowers had more petals than subsequent flowers (Fig. 4b). However, a significant increase total stamen number was observed, from 5.40 in the firstformed flower to 5.95 in flower 5 (Fig. 4c), which resulted from opposing trends in the number of lateral and medial stamens, with the former increasing to a greater extent than the decrease in the number of medial stamens (Fig. 4d, e). In addition to mean stamen number, approximately half (46.5\%) of wild type flowers possessed more than six and up to nine stamens. These supernumerary stamens were always medial, on either or both ab- or adaxial sides of the flower.

Primary or secondary erf12 inflorescences showed phyllotactic defects at almost complete penetrance $(98 \% ; \mathrm{N}=99$ inflorescences), resulting from an aberrant divergence angle of siliques on the stem, or inhibited internode elongation, to give clustered siliques (Fig. 31-n). These defects were maintained throughout inflorescence development and were not lost in later-initiated flowers (visible in Fig. 3n). 
Fig. 1 Phylogenetic relationship between Arabidopsis class VIIIa and VIIIb ERF/AP2 transcription factors and ERF12/MFS1 homologues from diverse plant taxa. The tree was compiled using the maximum likelihood method (PhyML programme), via the phylogeny.fr platform (Dereeper et al. 2008)

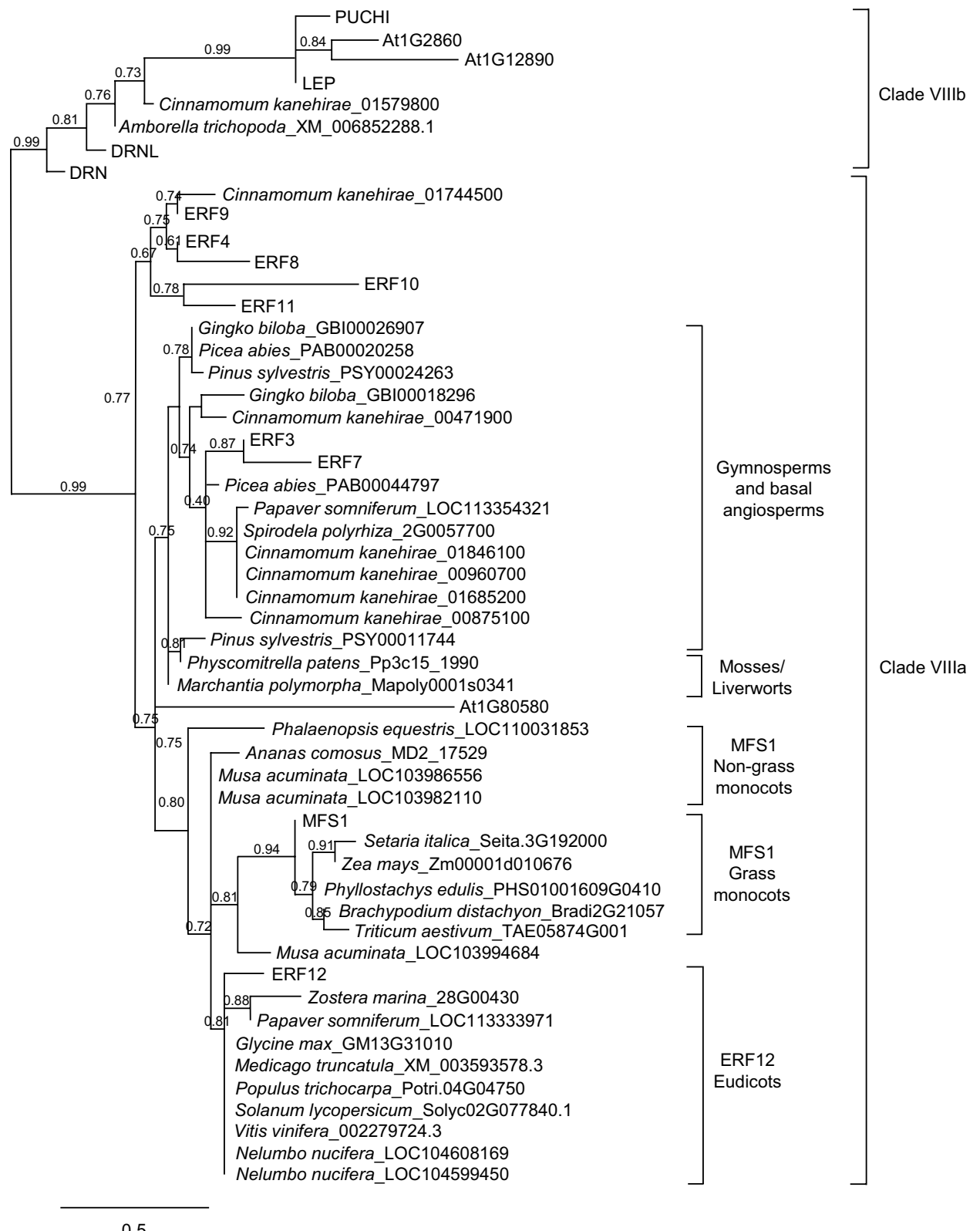

In controlled short-day growth conditions, the number of wild type and erf12 floral organs did not differ significantly (Fig. 4f for sepals).

\section{ERF12 is expressed dynamically in the IM and FM}

ERF 12::erGFP was dynamically expressed in the IM and FM during inflorescence development (Fig. 5a-c). Strongest expression was at the IM periphery at the border between newly initiated FMs from about P1 stage onwards, around the basal circumference of the floral primordium, as soon as the buttresses physically extended from the IM (Fig. 5d, e). In buds from about stage 3 onwards, foci of ERF 12::GFP expression marked the intersepal regions in floral buds and diffuse expression was present internally to the sepals throughout the FM (Fig. 5f, g) and in the tips of sepals as they overgrew the FM (Fig. 5h). Strong expression was also observed at the abaxial underside flank of the developing bud (Fig. 5i, j) and in guard cells of cotyledons and leaves (Fig. 5k, 1).

\section{Complementation of the erf12 mutant}

Sepal number in the first-formed flower was used to demonstrate complementation by genomic ERF12 sequences containing the ERF12 open reading frame and either the upstream genomic region $(g E R F 12 U p)$ or all upstream and downstream sequences ( $g$ ERF12UpDown). Five and 


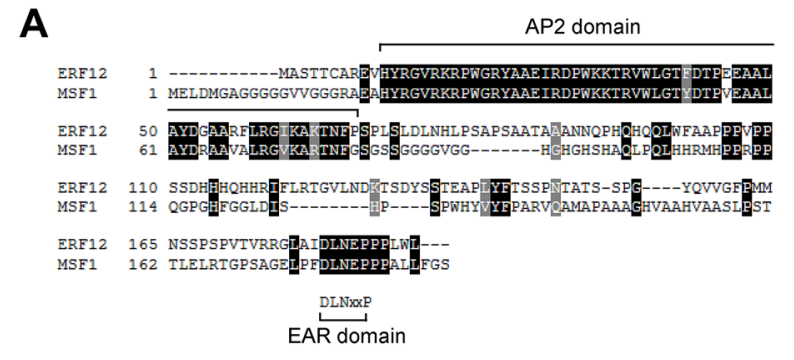

B

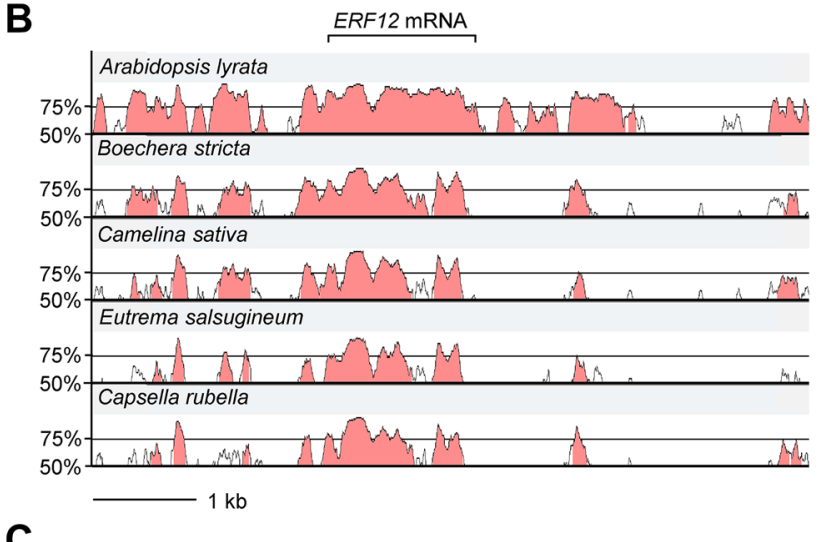

C
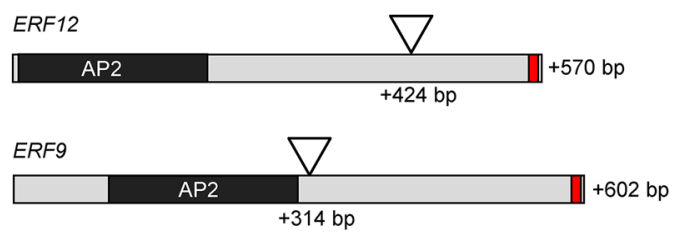

Fig. 2 Position of erf12 mutation and conservation of ERF12 protein domains and regulatory regions. Amino acid alignment of MFS1 from rice and Arabidopsis ERF12 (a) showing high conservation in the AP2 and EAR domains. VISTA blots of the ERF12 genomic locus, including the up- and downstream flanking genes compared between Arabidopsis thaliana and a range other Brassicaceae species (Arabidopsis lyrata, Boechera stricta, Camelina sativa, Eutrema salsugineum and Capsella rubella) (b). Schematic representation of the ERF9 and ERF12 open reading frames (c) showing the position of the T-DNA insertions and the encoded AP2 domain and EAR domain (red block)

seven independent transgenic lines containing $g E R F 12 U p$ or gERF12UpDown, respectively, all showed significantly statistically fewer sepals in the first-formed flower than erf 12 mutants (Fig. 6), mostly to the wild-type number of four. The late flowering erf 12 phenotype was not complemented (data not shown).

\section{Genetic interactions between erf12, drn, drnI and puchi}

The related AP2/ERF genes DRN, DRNL and $P U C H I$ redundantly regulate floral organ number in all whorls and $d r n$ drnl puchi mutants display trichomes at the base of floral pedicels at the position of the cryptic bract (Fig. 3o). We therefore investigated potential genetic interactions between these genes and ERF12 by creating a quadruple $d r n d r n l$ puchi erf12 mutant, which was not trivial, due to linkage between DRN, DRNL and ERF12 loci on chromosome 1. Similar to wild type, drn drnl puchi triple mutant flowers showed a gradient of organ numbers throughout inflorescence development, with a decrease in the number of trichomes subtending the pedicels, fewer sepals and petals and more stamens, from the first to the fifth flower (Fig. 7a-e). The erf 12 phenotype was not additive, synergistic or epistatic when combined with $d r n d r n l$ puchi. Instead, the frequency of trichomes subtending the first-formed flowers of drn drnl puchi erf 12 quadruple mutants was attenuated (Fig. 7e), and the number of floral organs in all whorls was significantly higher than in $d r n d r n l$ puchi triple mutants, although remained lower than wild type (Fig. 7a-d), except for the frequency of carpels, which was restored to the wild type number of two (Fig. 7d). Sepal number in the first few drn drnl puchi erf 12 flowers was significantly lower than in single erf 12 mutants. The penetrance of cotyledon defects in $d r n$ drnl puchi mutants was $46.16 \%(\mathrm{~N}=2110)$, and was attenuated in erf12 drn drnl puchi mutants to $30.94 \%$ $(\mathrm{N}=1939)$.

\section{erf12 enhances the ap2 mutant phentoype}

Because APETALA2 also functions in the floral transition and floral organ specification, we analysed the genetic interaction between ap2-7 and erf 12 mutants by creating a double ap 2-7 erf12 mutant. ap 2-7 mutant flowers consisted of carpelloid sepals and bract-like structures, some stamens and a relatively normal gynoecium (Fig. 8a, b). However, erf 12 enhanced the ap 2 mutant phenotype: stamens were absent in ap 2 erf 12 double mutant flowers and whorl one organs had a stronger carpeloid identity than in ap2 single mutants (Fig. 8c, d) and were often completely fused and resembled a carpel (Fig. 8e, f), with trichomes. Similar to erf 12 single mutants, the floral phyllotaxy of ap 2 erf 12 inflorescences was also defective (Fig. 8g), but was not more severe than that of single erf 12 mutants.

\section{Mutation of ERF9 does not affect floral development}

To characterise potential genetic redundancy between ERF 12 and other related AP2/clade VIIIa members, we analysed the phenotype of a T-DNA insertion mutant of ERF9 (Fig. 2c). erf9 showed wild type floral and inflorescence development (data not shown) and when combined

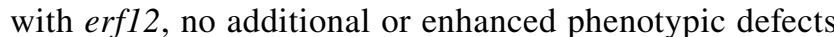
were observed in erf9 erf 12 plants to those of erf 12 single mutants. 

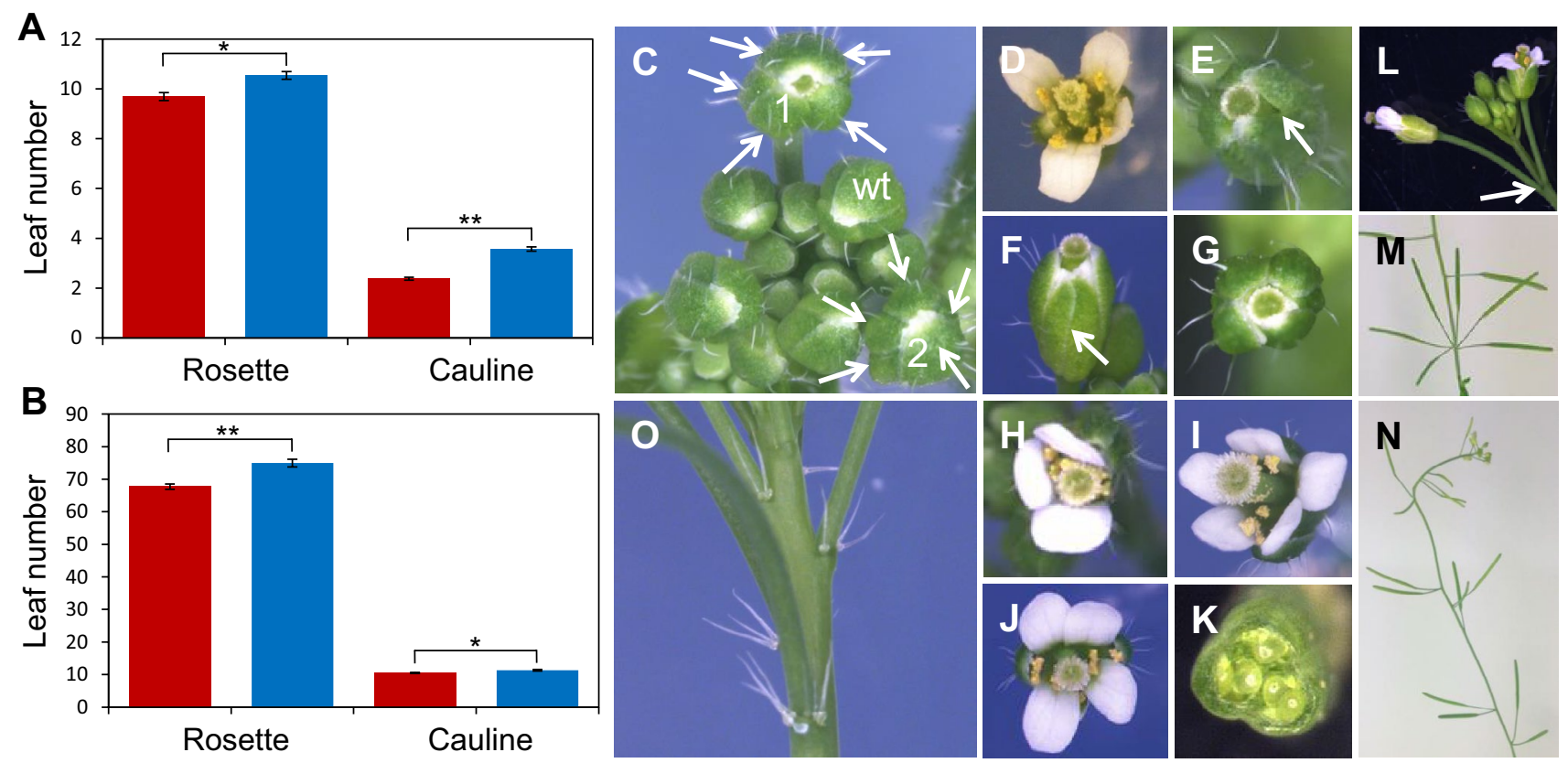

Fig. 3 erf12 mutant phenotype. Flowering time as indicated by rosette and cauline and leaf number for wild type (red bars) and erf 12 plants (blue bars) in long day (a) and short day (b) photoperiods; $* * p \leq 0.001 ; * * p \leq 0.0001$. Sepal phenotypes of erf12 mutants: topview of young inflorescences (c) showing supernumerary sepals (all sepals marked by arrows) in the first two flowers (marked 1 and 2). A wild-type bud with four sepals is marked "wt". A wild-type flower with four petals and six stamens clearly visible (d). Single buds showing fused supernumerary abaxial sepals (e; fusion marked with

\section{Discussion}

\section{ERF12 and MFS1 are an angiosperm-specific invention}

The MFS1 AP2 protein in rice, which determines floral meristem and organ identity, prompted a phylogenetic analysis, which identified the Arabidopsis orthologue as ERF12. The absence of ERF12/MFS1 homologues in plant radiations prior to the emergence of angiosperms and in Amborella trichopoda, the monotypic sister genus to all angiosperms, suggests that ERF12-type proteins represent a derived angiosperm trait subsequent to the divergence of eudicots from basal angiosperms that co-evolved with the diversification of angiosperm floral morphology. This contrasts with close AP2/ERF relatives belonging to clade VIIIb, which are more ancestral (Chandler 2018). This conclusion is supported by several other pieces of evidence. Firstly, no ERF12/MFS1 orthologue is present in the three gymnosperm genomes compared, nor in that of the magnoliid Cinnamomum kanehirae, another basal angiosperm, whose closest protein relatives group in the ERF3/7 clade. The robust and comprehensive analysis of plant taxa across plant kingdom (Fig. 1) show that an arrows) or supernumerary sepals at various positions (f, g). erf12 flowers with three (h) or five (i) petals; seven stamens (j) and three fused carpels (k). Floral phyllotaxy defects in inflorescences of erf12 mutants: the first two flowers occupying the same stem node separated by an acute angle (l), or clustering of flowers due to aberrant phyllotaxy and internode elongation $(\mathbf{m}, \mathbf{n})$. Trichomes at the position of the cryptic bract, subtending floral pedicels of $d r n d r n l$ puchi mutants (o)

ERF12/MFS1 homologue is clearly shared by all early non-grass monocots analysed (Ananas comosum, Musa acuminata, Phalaenopsis equisetum, Spirodela polyrhiza, Zostera marina), and in all grass monocots and evolutionarily young eudicots analysed. Furthermore, the closest Amborella relative does contain an ESR domain, confirming that it belongs to the clade VIIIb proteins (Chandler 2018). Another argument is that ERF12 predominantly affects sepals, which are a relatively recent morphological innovation, since they are absent in Amborella, the best extant reference for understanding the evolutionary molecular genetic basis of flower development (Amborella Genome Project 2013) and also the magnoliid Cinnamomum kanehirae. The tepals of Amborella flowers show a gradual morphological transition from outer to inner floral organs and floral trait modelling suggests that flowers of the most recent angiosperm ancestor had an undifferentiated perianth consisting of multiple tepals (Sauquet et al. 2017). Phalaenopsis equestris also possesses tepals, but contains a protein that more closely aligns with ERF12 than other group VIIIa proteins (Fig. 1). This might represent an intermediate ancestor in the evolution of an ERF12 protein associated with sepal development and flowers having a characteristic angiosperm floral body 
A
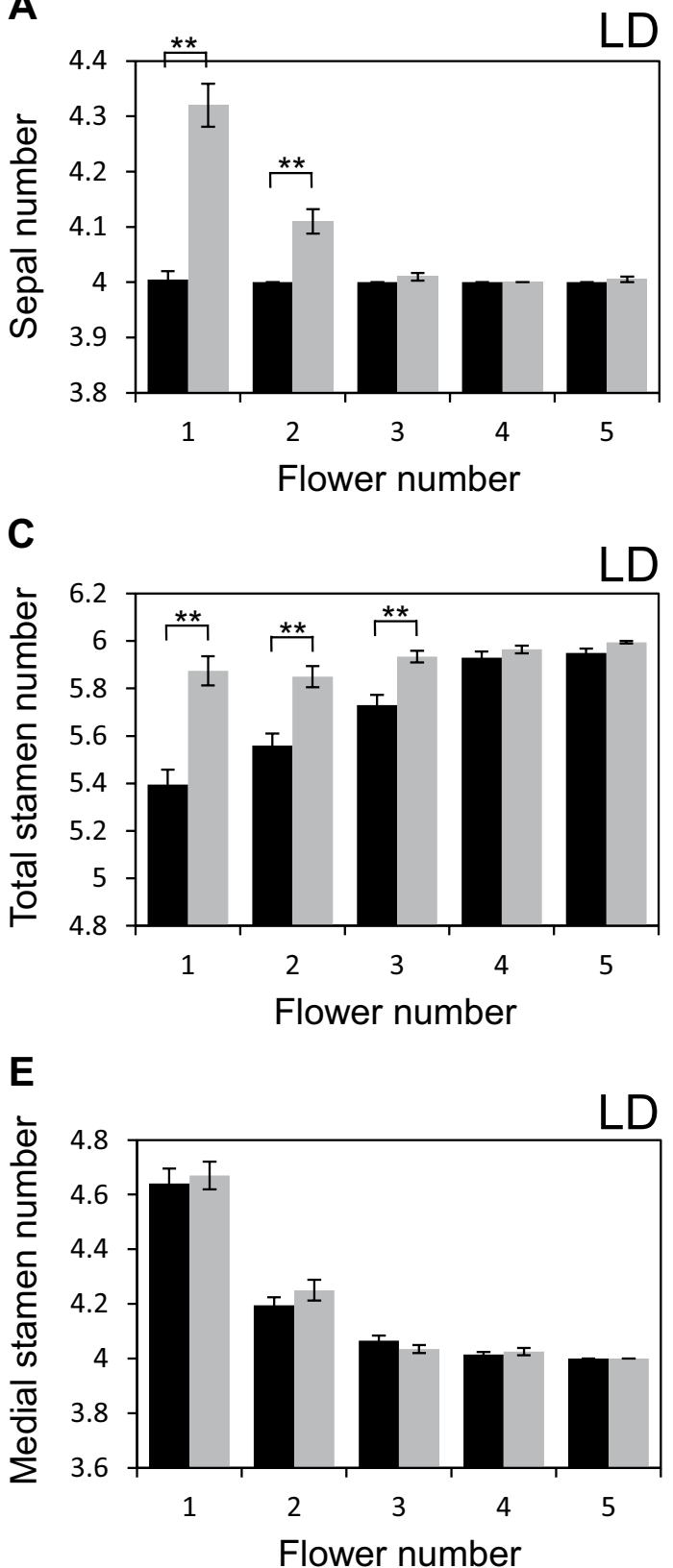

Fig. 4 Sepal, petal and stamen number is labile in earliest-formed wild type and erf12 flowers. Frequency of sepals (a); petals (b); total stamens (c); lateral stamens (d); medial stamens (e) in the first five flowers of wild type and erf12 mutants grown in long days. $\mathrm{N}=200$; $* p<0.05 ; * * p<0.0001$. Frequency of sepals in the first five flowers

plan. Widespread whole-genome duplication across angiosperms, leading to a diversification in gene family content, particularly in crucifers (Amborella Genome Project 2013), probably led to the coopting and neofunctionalisation of $E R F$ genes such as $E R F 12$ for specific floral functions. During angiosperm evolution, perianth differentiation is thought to have multiple independent origins and many theories exist concerning the gene neo- and
B

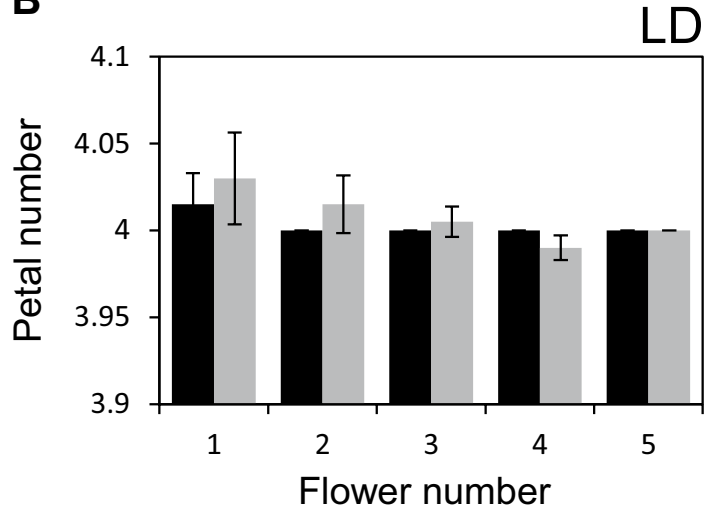

D

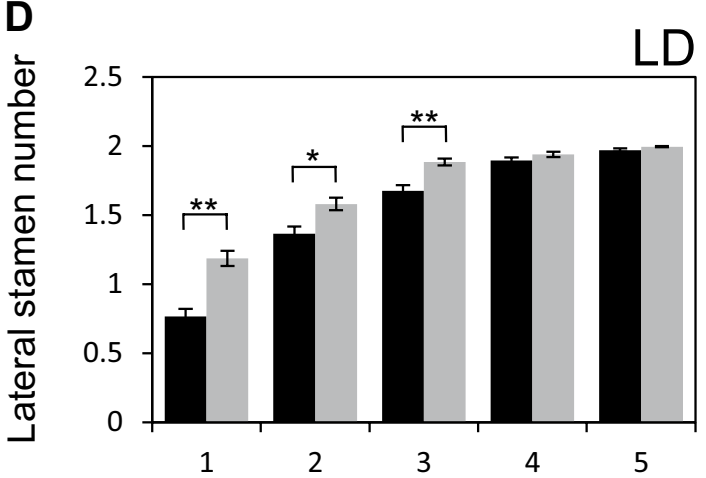

Flower number

$\mathbf{F}$

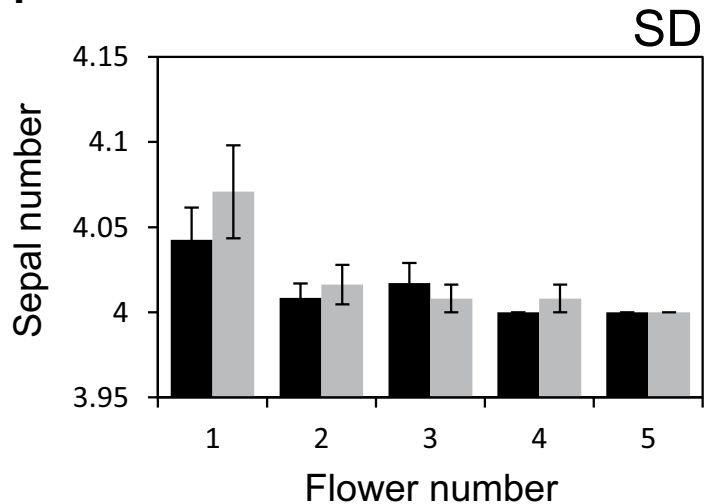

of wild type and erf12 mutants grown in short days $(\mathrm{F})(\mathrm{n}=117$ for wild type; 123 for erf12). Black and grey columns represent wild type and erf12, respectively. $L D$ long days, $S D$ short days. Flower number 1 represents the first-formed flower

subfunctionalisations that underlay these events (Irish 2009). One example is the conversion of tepals into sepals into Phalaenopsis equestris brought about by silencing SEPALLATA3 (Pan et al. 2014). However, ERF12 function is not associated with perianth organ identity, but organ merosity, and the phylogenetic analysis here that exploits the currently available sequenced genomes representing key plant evolutionary stages, robustly supports 

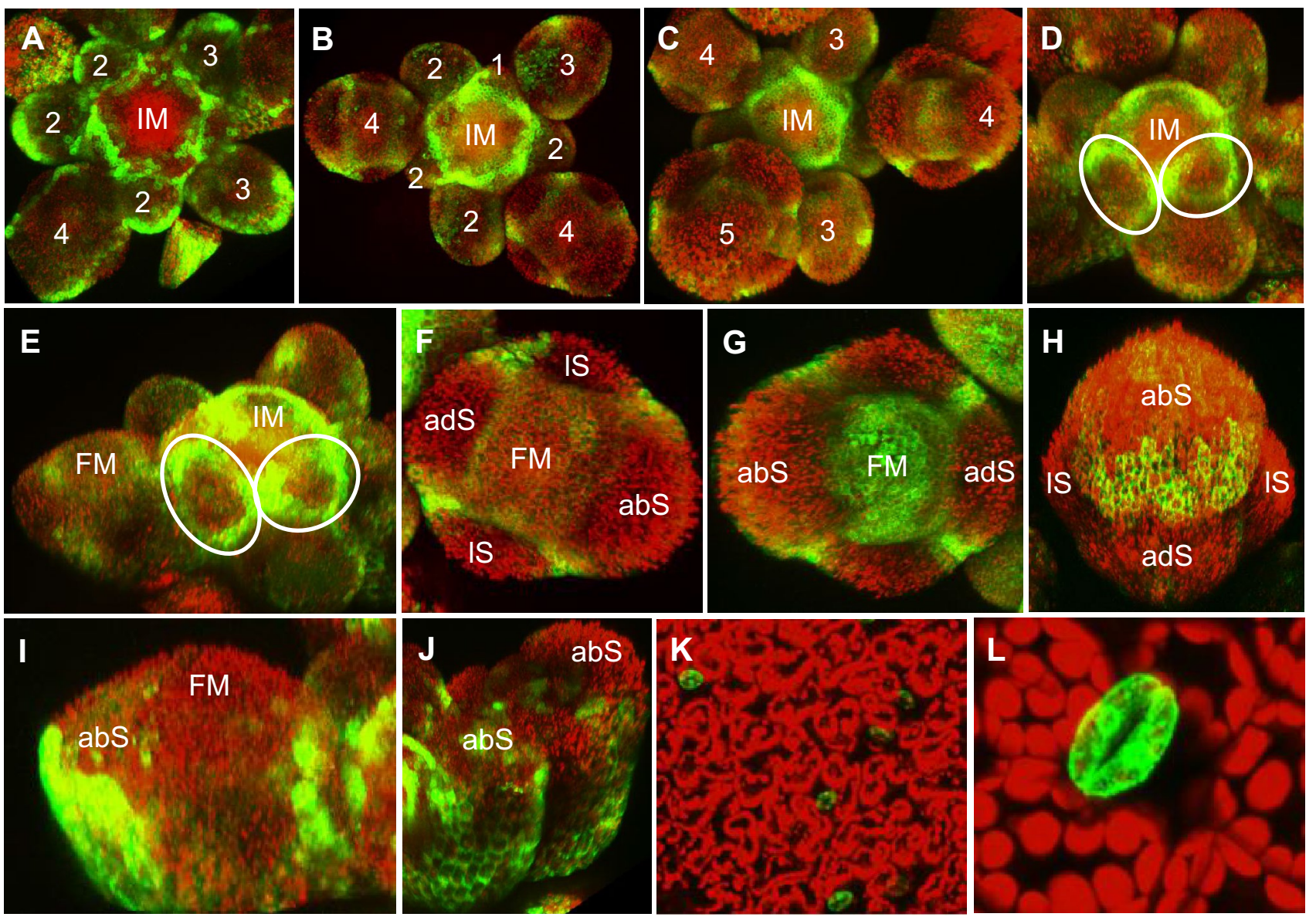

Fig. 5 ERF12 is dynamically expressed in developing flowers and in guard cells. Expression of pERF12::erGFP in top views of inflorescence apices from independent transgenic lines showing flowers in different stages of development $(\mathbf{a}-\mathbf{c})$; oblique views of inflorescence apices showing rings of $p E R F 12::$ erGFP expression surrounding the emerging floral primordia (marked by oblongs) (d, e); top views of

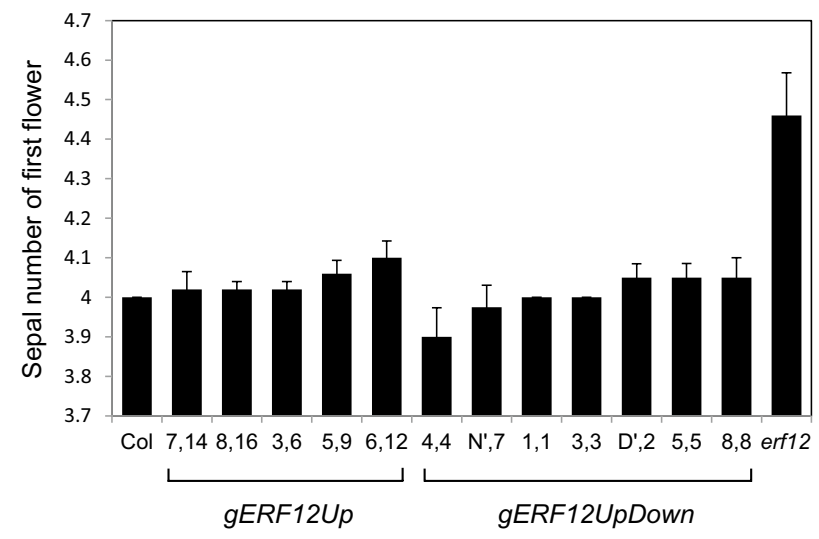

Fig. 6 Complementation of the erf12 mutant. Sepal number in the first-formed flower of multiple independent erf12 transgenic lines complemented with the ERF12 locus containing upstream ( $g E R$ $F 12 U p$ ) or up- and downstream regulatory sequences ( $g E R F 12 U p$ Down) early (f) and late stage 4 (g) and stage 6 (h) floral buds; saggital view of stage 4 floral buds (i, j); epidermis of a cotyledon (k); a leaf (l). IM inflorescence meristem, $F M$ floral meristem, $a b S$ abaxial sepal, $a d S$ adaxial sepal, $l S$ lateral sepal; numbers represent floral stages according to Smyth et al. (1990)

the hypothesis that ERF12 neofunctionalised comcomitant with the appearance of angiosperm flowers with a differentiated perianth.

In Arabidopsis, clade VIIIa AP2/ERF members except ERF8 and ERF12, phylogenetically associate as sister pairs or paralogues (ERF3 and ERF7; ERF4 and ERF9; ERF10 and ERF11). The absence of redundant paralogues might explain why single erf 12 mutants display a phenotype, whereas redundancy between ERF9 and ERF4 might mask erf9 phenotypes. Few clade VIIIa ERF proteins have been functionally characterised, but ERF11, which is encoded by the adjacent locus to ERF12, promotes internode elongation via gibberellin synthesis (Zhou et al. 2016) and represses ethylene synthesis ( $\mathrm{Li}$ et al. 2011). ERF9 is involved in plant defence against necrotic fungi via ethylene/JA pathways (Maruyama et al. 2013) and ERF8 is a transcriptional repressor that negatively 

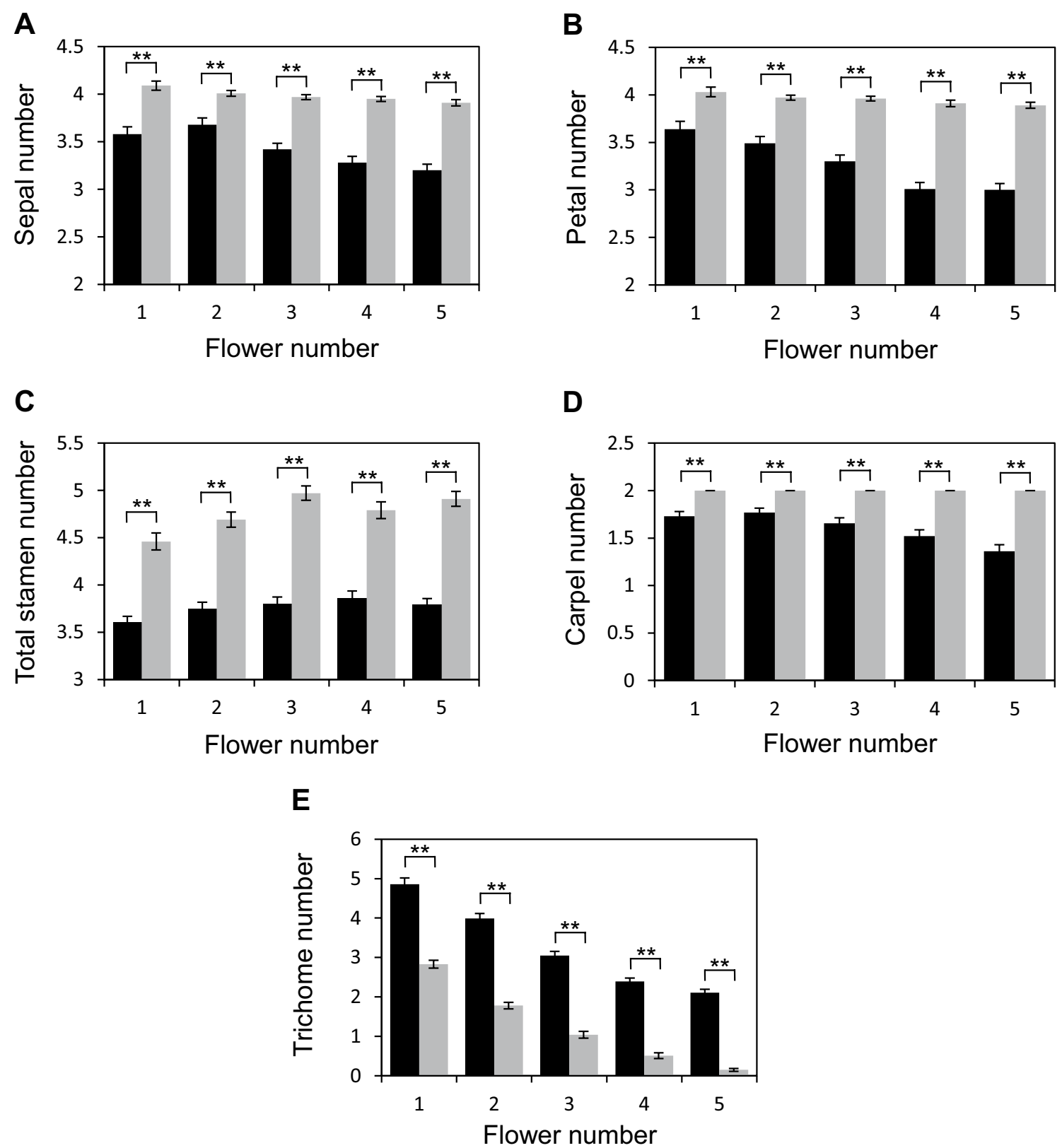

Fig. 7 ERF12 interacts genetically with related AP2/ERF genes DORNRÖSCHEN (DRN), DORNRÖSCHEN-LIKE (DRNL) and $P U C H I$. Frequency of sepals (a); petals (b); total stamens (c); carpels (d): trichomes subtending the pedicels (e) in drn drnl puchi and erf12

regulates $\mathrm{ABA}$-mediated responses and is involved in immune signalling (Cao et al. 2018) and water stress in kiwifruit (Zhang et al. 2017). Although ERF12 has been functionally implicated in diverse hormone pathways, such as auxin (Lewis et al. 2013), gibberellin (Cao et al. 2006), salicylic acid and methyl jasmonate (Caarls et al. 2017), the data here implicate it as the first ERF VIIIa clade member with a developmental role. drn drnl puchi mutants. Black and grey columns represent drn drnl puchi and erf12 drn drnl puchi mutants, respectively. Flower number 1 represents the first-formed flower. $\mathrm{N}=200 ; * * p \leq 0.0001$

\section{Stereotypy in wild type floral organ number is initially unstable}

Stochastic variation in floral organ number exists within populations or individuals of many species. For example, petal number in Cardamine hirsuta (Pieper et al. 2016) is naturally variable and together with stamen number, is regulated by seasonal temperature (Matsuhashi et al. 2012; McKim et al. 2017) and tepal number varies among the 
Fig. 8 erf12 enhances the ap2 phenotype. An exemplary ap 2 flower (a) and inflorescence (b). Flowers of ap 2 erf 12 plants (c, d). Inflorescences of ap 2 erf 12 plants $(\mathbf{e}-\mathbf{g})$. Note the complete fusion of outer whorl organs (arrows) in (e) and the aberrant phyllotaxy (arrow) in (g)
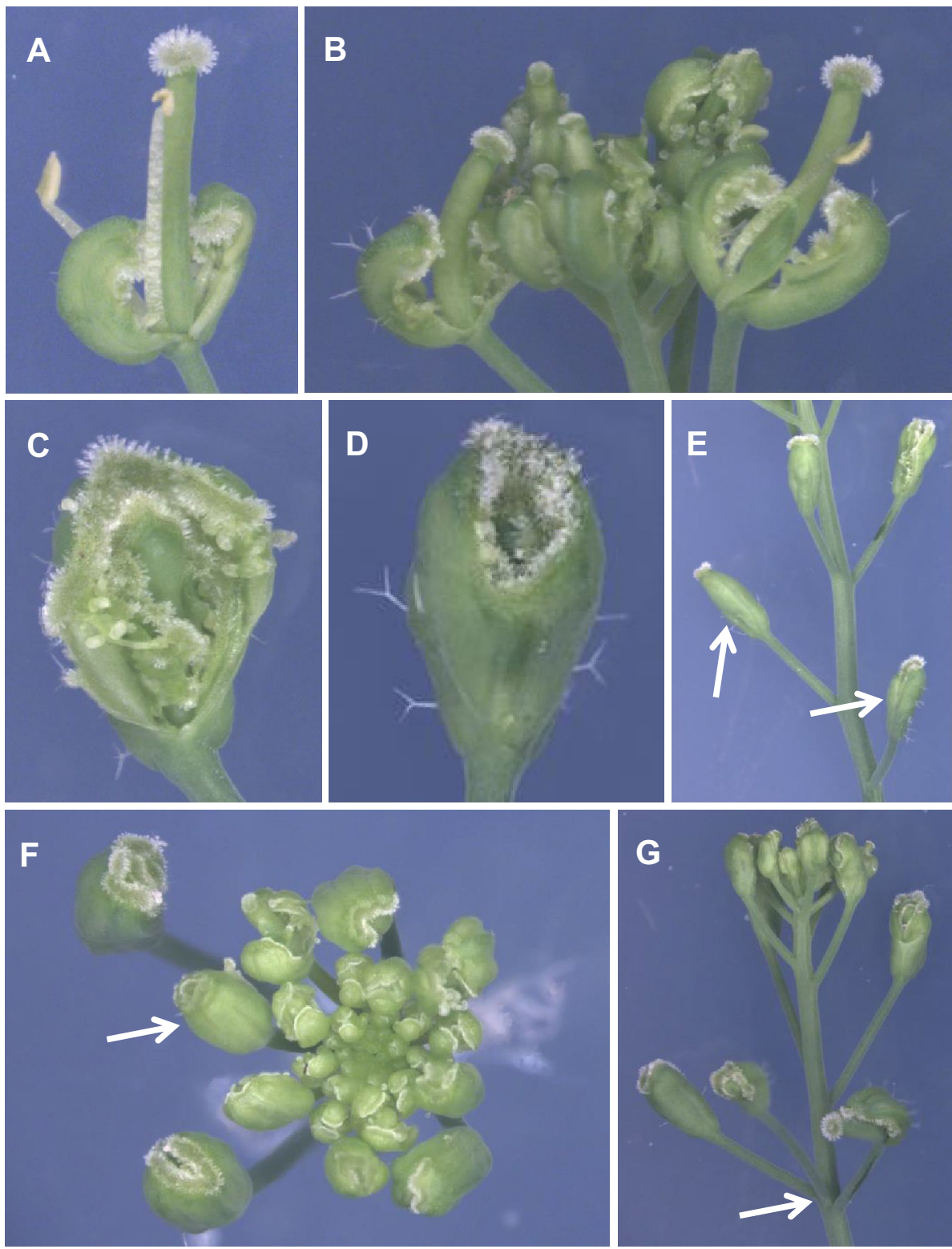

Ranunculaceae (Kitazawa and Fujimoto 2014). Stamens are numerically the most variable Arabidopsis organ, due to the frequent absence of lateral stamens (Anderson and Roberts 1998). In addition to an environmental and quantitative genetic basis for organ number variation, we show here that the number of wild type floral organs, especially of stamens, is initially developmentally labile and stabilises after the first few flowers. The floral transition thus involves variability in organ merosity, as well as meristem identity changes. The timing of stamen founder cell specification is unknown; auxin response maxima have been spatially associated with early developmental time points, but the founder-cell marker $D R N L$ is expressed prior to DR5 (Chandler et al. 2011) and non-robust positional information might result from the imprecise resolution of initially diffuse expression domains of positional regulators. Floral organ number and increased organ fusion and homeosis in wild-type Arabidopsis are enhanced by GA treatment (Plackett et al. 2018) and the most common homeotic conversions are petalloid stamens (Chandler and Werr 2011). Variable organ numbers in first-formed wild type flowers probably represents variable molecular stochasticity in expression levels or boundaries of homeotic or other regulatory genes (Kitazawa and Fujimoto 2014), especially considering the close proximity of petal and lateral stamen founder cell populations (Chandler et al. 2011). 


\section{ERF12 promotes the floral transition and floral meristem identity}

The late-flowering phenotype of erf12 mutants in LD and SD establishes that ERF 12 promotes the IM-to-FM transition independently of photoperiod. This contrasts with mutation of several Arabidopsis AP2-type genes that repress the floral transition, including $A P 2$ (Ohto et al. 2005), SCHLAFMÜTZE (Mathieu et al. 2009), TARGET OF EAT1 (TOE1) and TOE2 (Aukerman and Sakai 2003), and GLOSSY15 in maize (Lauter et al. 2005). More cauline leaves in erf12 than wild type in both photoperiods represents an extension of the meristem maturity phase, where phytomers transition from containing a secondary inflorescence meristem in the axil of a cauline leaf, to a floral meristem in the axil of a cryptic bract (Park et al. 2014). Despite repressing the floral transition, other AP2-type genes promote FM identity and simultaneously repress vegetative characteristics from flowers. Thus, sepals of Arabidopsis ap2 mutants and lip1 lip2 double mutants in Antirrhinum are often converted to leaf- or bract-like structures (Bowman et al. 1989; Keck et al. 2003) and supernumerary bracts in rice and double indeterminate spikelet $1 /$ sister of indeterminate spikelet 1 in maize display bracts instead of flowers (Lee et al. 2007; Chuck et al. 2008). Additionally, mutation of the Arabidopsis AP2/ERF gene PUCHI results in partial bract outgrowth (Karim et al. 2009), and of its orthologues FRIZZY PANICLE in rice (Komatsu et al. 2003) and BRANCHED SILKLESS in maize (Colombo et al. 1998), blocks the transition from spikelet to floral meristem identity. These mutant phenotypes can be interpreted as heterochronic effects, and the delayed or abolished transition from spikelet meristem or IM to FM produces ectopic bracts or leaf-like organs. The concept of the floral transition as a multidimensional process involving different spatiotemporal components is underlined by uncoupling of the floral transition and bolting by non-permissive conditions (Pouteau and Albertini 2011), which also causes the floral reversion of incompletely committed meristems in many species (Asbe et al. 2015). In Petunia, floral reversion has revealed a continuum of variation at the levels of meristem identity, primordium initiation and floral organ identity (Pouteau et al. 1998). Similarly, Arabidopsis puchi flowers partially spontaneously revert (Karim et al. 2009). Flower development is also a multistep process that invokes dynamic and competing ab-/adaxial polarity or centroradial models (Chandler and Werr 2014). Based on transcriptome analysis in pea where the ERF12 homologue is expressed in the vegetative SAM (Wong et al. 2008), by analogy, ERF 12 might be present in the vegetative Arabidopsis meristem, raising questions whether it is upregulated in the IM either due to the endogenous age-related developmental programme or on the floral transition, and if this were the case, threshold models for its function could be invoked, or its functional specificity might depend on appropriately expressed interaction partners.

Sepal initiation occurs independently of a stem cell population as marked by CLAVATA3 (CLV3) and WUSCHEL (WUS), which are expressed in IM stem-cells and in the FM centre from late floral stage 2, but are absent during early FM development (Goldshmidt et al. 2008; Yadav et al. 2009). We speculate that in addition to a delayed floral transition, erf 12 mutants have an extended meristem maturation phase in which FM identity and autonomy are irreversibly acquired, and thus a prolonged sepal initiation phase prior to the centroradial initiation of inner floral organs, leading to more sepals. Genes such as $A P 1$ and $A P 2$ that regulate FM identity in Arabidopsis, are also required for sepal identity (Litt 2007), presumably because FM identity is acquired during the sepal initiation phase, which involves unidirectional polarity (Chandler and Werr 2014). The stochastic variation in wild-type floral inner organs is enhanced in erf12, suggesting that $E R F 12$ also contributes to organ merosity during the centroradial phase of inner organ initiation. Although erf12 shows photoperiod-independent late-flowering, the increased frequency of sepals, petals and lateral stamens in erf12 is dependent on long-days. This might reflect the photoperiodic regulation of ERF12 transcription (Mantegazza et al. 2014), or be because short-day flowering as a default pathway, is potentially more robust than the long-day promotion of flowering, which involves the activation and more precise convergence of pathways at a temporally specific point and might be thus more inherently unstable and prone to disruption.

Phyllotaxis at the apical meristem is largely regulated by auxin response maxima (Reinhardt et al. 2000); however, disrupted phyllotaxy can also result from meristematic effects that cause delayed floral primordium outgrowth, as in arabidopsis histidine phosphotransfer protein 6 mutants (Besnard et al. 2014), or post-meristematic mechanisms that perturb internode elongation due to the inappropriate regulation of CUP-SHAPED COTYLEDON (CUC) 2 by miRNA164 (Peaucelle et al. 2007), as observed in higherorder cuc mutants (Burian et al. 2015). The basis of clustered siliques and disrupted phyllotaxis in erf 12 inflorescences is unknown, but appears at least partly to result from aberrant internode elongation.

\section{Aspects of the erf12 mutant phenotype are complemented by ERF12}

Supernumerary sepals in the first-formed flower was used as the most easily quantifiable phenotype to assess erf 12 complementation by the genomic ERF12 locus. Transformation by $g E R F 12 U p$ was sufficient to complement the erf 12 sepal phenotype. This might reflect the higher sequence conservation revealed by phylogenetic shadowing in the upstream 
promoter region compared to downstream sequences. However, the late flowering phenotype of erf12 was not complemented by the whole genomic sequence between the up- and downstream flanking genes, potentially reflecting the importance of the appropriate physical open chromatin context of ERF 12 within its native chromosomal environment, since epigenetic mechanisms are also major components of flowering time regulation (Ahmad et al. 2010).

\section{ERF12 expression spatiotemporally coincides with the observed mutant phenotypes}

Dynamic $p E R F 12::$ erGFP expression in the IM and FMs throughout inflorescence development and not only in the first-formed flowers where the strongest floral mutant phenotypes were observed, suggests that these phenotypes also depend on the developmentally regulated expression of downstream targets. The ring of $p E R F 12::$ erGFP expression at the base of young floral primordia as they emerge from the IM might mechanistically underlie a function for ERF12 in the timing of primordium outgrowth and establishing phyllotaxy. Strong $p E R F 12::$ erGFP expression in the abaxial side of floral stage 1 and 2 FMs, during the sepal initiation phase coincide with the erf12 sepal phenotype and strong foci of pERF 12::erGFP expression in the intersepal regions might be the basis for sepal fusion, and expression throughout the FM encompasses the sites of stamen and petal founder cells (Chandler et al. 2011). In summary, pERF12 expression data spatiotemporally coincide with all aspects of the mutant phenotypes. Furthermore, ERF 12 expression in cotyledon and leaf guard cells shows that the gene is active in embryonic and postembryonic tissue in differentiated cell types as well as in meristematic tissue.

\section{ERF12 and MFS1 are functionally similar}

A comparison between the functions of $E R F 12$ and rice MFS1 must consider the different floral morphologies between both species. Wild-type rice spikelets contain two rudimentary glumes, two sterile lemmas, in addition to a terminal floret with a single lemma and palea in whorl 1, two lodicules in whorl 2, six stamens in whorl 3, and one carpel in whorl 4. The lodicules are considered by some to be modified petals (Whipple et al. 2007). The lemma and palea have historically been interpreted as extra-floral organs, as a bract and prophyll, respectively (Lombardo and Yoshida 2015). However, because they express floral genes and based on rice floral homeotic mutants, they are probably sepal analogues (reviewed in Lombardo and Yoshida 2015), with the palea potentially representing a differentiated lemma (Ambrose et al. 2000). In $m f s 1$ mutants, the sterile lemma is homeotically converted into the rudimentary glume and the majority of $m f s l$ spikelets contain an extra lemma-like organ, often two degenerated palea-like organs instead of one, and a variable number of stamens (Ren et al. 2013). Furthermore, an enlarged FM suggests that spikelets with an extra lemma might derive from two florets, due to a delay in FM determinacy acquisition. Thus, the pleiotropic $m f s 1$ phenotype consists of three components: a delayed spikelet meristem to FM transition, organ homeoses (sterile lemma/ glume and palea) and increased organ merosity (supernumerary lemmas and paleae). Despite the absence of intermediate meristems in Arabidopsis equivalent to the rice spikelet meristem, delayed flowering of erf12 mutants represents a similar delay in establishing FM identity to $m f s l$. No organ homeoses were observed in erf12, but this aspect of the $m f_{s} 1$ phenotype is probably masked, because in contrast to lemma and palea, Arabidopsis sepals are morphologically similar and no analogues of sterile lemmae or rudimentary glumes are present. Conversely, rice has no analogous organs to petals, but the variable number of stamens and sepals in erf12 phenocopies labile numbers of stamens, lemmas and paleae in $m f s 1$. The largely congruent $\operatorname{erf} 12$ and $m f s 1$ mutant phenotypes suggests that MFS1 and ERF12 are functionally equivalent, which is further supported by the high conservation of the AP2 domain and the shared C-terminal EAR-like motif. The erf12 phenotype manifests within a narrow developmental time-window including the first-formed flowers, which suggests a heterochronic function for ERF12; but no evidence is available concerning changes in the severity of the $m f s 1$ phenotype along the rachis, the timing of the floral transition or defects in phyllotaxy.

\section{ERF12 interacts genetically with closely related $E R F$ factors and $A P 2$}

Genetic interactions between $E R F 12$ and its closely related ERF clade VIIIb genes DRN, DRNL and PUCHI were revealed by higher-order mutant analysis. Loss of ERF12 function in the $d r n d r n l$ puchi triple mutant background led to attenuated phenotypes, suggesting that the phenotypic severity of $d r n d r n l$ puchi plants requires ERF 12 function. Conversely, the supernumerary sepal phenotype of erf12 single mutants was counteracted by loss of $D R N$, DRNL and PUCHI function. Thus, ERF12 and the combined functions of DRN,DRNL and PUCHI are opposing in terms of the repression and promotion of organ initiation, respectively. DRN, DRNL and PUCHI are considered to be transcriptional activators (see Chandler 2018) and all contain a transcriptionally active ESR domain (Nomura et al. 2009). Conversely, ERF12 contains a C-terminal EAR-like repressor domain, which might transcriptionally repress downstream targets at the timepoint of incipient FM development within the IM, which is associated with large-scale gene downregulation (Wellmer et al. 2006). ERF12 also contains an AP2 transcriptional activation domain that might 
facilitate protein-protein interactions (Chandler et al. 2007); thus, ERF12 might activate and repress distinct subsets of downstream targets, similar, to AP2 (Yant et al. 2010). The genetic interactions between ERF12 and DRN,DRNL and $P U C H I$ might represent the combined outread of cumulative activated and repressed targets. Although scant detailed knowledge concerning direct gene targets for these genes currently limits further interpretation of the interactions, it demonstrates that all genes converge on similar downstream targets. In rice, $M F S 1$ transcriptionally activates the $A P 2$ homologues SNB and OSIDS1 (Ren et al. 2013). Here, the ap2 phenotype is dependent on ERF12 and is enhanced by erf12. In strong ap 2 alleles, sepals are converted into carpels, petals are absent and the number of stamens is reduced, whereas erf12 ap2 mutants only produce carpels. This synergistic phenotype is similar to that of ap2 ant double mutants, which only produce carpels (Elliott et al. 1996). This potentially reflects the regulation of $A P 2$ transcription or its expression domain by ERF12. AP2 possesses a cadastral role in regulating AGAMOUS expression (Drews et al. 1991) and potentially loss of $A P 2$ and ERF12 function regulate downstream B-function genes to abolish stamens.

The absence of observable mutant phenotypes for $\operatorname{erf} 9$ or genetic interactions in combination with erf 12 suggests that either it is not involved in development, or functions redundantly with other related $E R F$ genes, which requires confirmation via a more systematic higher order mutant analysis of group VIIIa members.

In summary, we have characterised pleiotropic roles of the ERF12 transcription factor in reproductive development (Fig. 9): firstly, it promotes the floral transition and specifies FM identity; secondly, dependent on long-day photoperiods, it represses floral organ initiation, particularly of sepals and stamens; supernumerary erf12 sepals potentially arise from an extension of the FM maturity phase. Thirdly, as a putative repressor, ERF12 counteracts the promotion of floral organ initiation by the combined activities of the related transcription factors DRN, DRNL and PUCHI and stabilises the inherent lability of organ number of the firstformed wild type flowers. Fourthly, ERF12 converges with the AP2 pathway to specify organ identity. Finally, ERF12 is continuously required in the IM to maintain appropriate FM phyllotaxy. Alternatively, we can also speculate that the observed erf 12 phenotypes could result from the loss of a wild-type function that represses activators of the floral transition or growth, such that in the mutant, later flowering and overgrowth, including supernumerary organs arising under certain physiological conditions. This study is confined to a single mutant allele (also for erf9), because no others are currently available in public stock centres but the caveat exists that we cannot completely exclude that these alleles are null, especially erf 12 , because the T-DNA insertion is in the $3^{\prime}$ region of the open reading frame. However, the

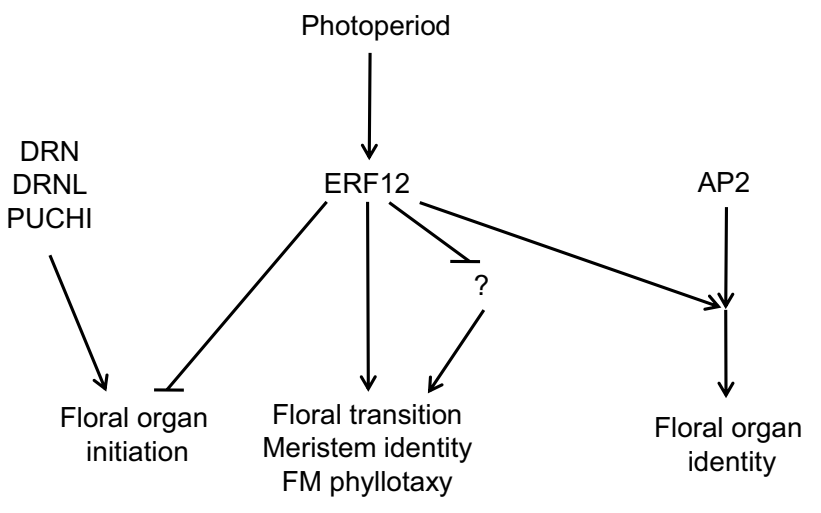

Fig. 9 Model for the integration of ERF12 function into other genetic pathways and developmental processes. ERF12 function is dependent on photoperiod and contributes pleiotropic roles to floral organ initiation and floral meristem identity. ERF12 is a putative transcriptional repressor and opposes the functions of the related AP2 transcription factors DORNRÖSCHEN (DRN), DORNRÖSCHEN-LIKE (DRNL) and PUCHI, and integrates into the APETAL2 (AP2) pathway to regulate floral organ identity. Alternatively, ERF12 might repress unknown growth or floral transition activators, leading to late flowering and organ overproliferation when this repressive function is lost in the mutant

successful complementation and conserved functions with rice MFS1 convincing and unequivocally consolidate the interpretations and conclusions concerning ERF12 functions in floral organogenesis. The discovery of a novel gene function that contributes to phase change and floral organogenesis suggests that the genetic network involved in these processes might not yet be fully elucidated, even at a nonredundant genetic level. ERF12 also enhances our understanding concerning the incremental processes that comprise the floral transition, and the stochastic instability in wildtype floral organ merosity reveals that the floral transition also focuses positional information required to appropriately generate floral organ founder cells in the FM, in addition to establishing meristem identity.

Acknowledgements This work was supported by the Deutsche Forschungsgemeinschaft via Grant WE1262/11-2 to W. Werr.

Author contributions Experiments were designed and conducted by JWC. The data were analysed by JWC and the manuscript was written by JWC and edited by JWC and WW. All authors read and approved and final manuscript.

Open Access This article is distributed under the terms of the Creative Commons Attribution 4.0 International License (http://creativeco mmons.org/licenses/by/4.0/), which permits unrestricted use, distribution, and reproduction in any medium, provided you give appropriate credit to the original author(s) and the source, provide a link to the Creative Commons license, and indicate if changes were made. 


\section{References}

Ahmad A, Zhang Y, Cao XF (2010) Decoding the epigenetic language of plant development. Mol Plant 3:719-728

Amborella Genome Project (2013) The Amborella genome and the evolution of flowering plants. Science 342:1241089

Ambrose BA, Lerner DR, Ciceri P, Padilla CM, Yanofsky MF, Schmidt RJ (2000) Molecular and genetic analyses of the silkyl gene reveal conservation in floral organ specification between eudicots and monocots. Mol Cell 5:569-579

Anderson ML, Roberts JA (1998) Arabidopsis. CRC Press, Boca Raton, p 407

Asbe A, Matsushita SC, Gordon S, Kirkpatrick HE, Madlung A (2015) Floral reversion in Arabidopsis suecica is correlated with the onset of flowering and meristem transitioning. PLoS ONE 10:e127897

Aukerman MJ, Sakai H (2003) Regulation of flowering time and floral organ identity by a microRNA and its APETALA2-like target genes. Plant Cell 15:2730-2741

Besnard F, Refahi Y, Morin V, Marteaux B, Brunoud G, Chambrier P, Rozier F, Mirabet V, Legrand J, Lainé S et al (2014) Cytokinin signalling inhibitory fields provide robustness to phyllotaxis. Nature 505:417-421

Blázquez MA, Soowal LN, Lee I, Weigel D (1997) LEAFY expression and flower initiation in Arabidopsis. Development 124:3835-3844

Bowman JL, Smyth DR, Meyerowitz EM (1989) Genes directing flower development in Arabidopsis. Plant Cell 1:37-52

Burian A, Raczyńska-Szajgin M, Borowska-Wykręt D, Piatek A, Aida M, Kwiatkowska D (2015) The CUP-SHAPED COTYLEDON2 and 3 genes have a post-meristematic effect on Arabidopsis thaliana phyllotaxis. Ann Bot 115:807-820

Caarls L, Van der Does D, Hickman R, Jansen W, Verk MC, Proietti S, Lorenzo O, Solano R, Pieterse CM, Van Wees SC (2017) Assessing the role of ETHYLENE RESPONSE FACTOR transcriptional repressors in salicylic acid-mediated suppression of jasmonic acid-responsive genes. Plant Cell Physiol 58:266-278

Cao D, Cheng H, Wu W, Soo HM, Peng J (2006) Gibberellin mobilizes distinct DELLA-dependent transcriptomes to regulate seed germination and floral development in Arabidopsis. Plant Physiol 2:509-525

Cao FY, DeFalco TA, Moeder W, Li B, Gong Y, Liu XM, Taniguchi M, Lumba S, Toh S, Shan L, Ellis B, Desveaux D, Yoshioka K (2018) Arabidopsis ETHYLENE RESPONSE FACTOR 8 (ERF8) has dual functions in ABA signaling and immunity. BMC Plant Biol 18:211

Chandler JW (2018) Class VIIIb APETALA2 ethylene response factors in plant development. Trends Plant Sci 23:151-162

Chandler JW, Werr W (2011) The role of DORNRÖSCHEN-LIKE in early floral organogenesis. Plant Signal Behav 6:1244-1246

Chandler JW, Werr W (2014) Arabidopsis floral phytomer development: auxin response relative to biphasic modes of organ initiation. J Exp Bot 65:3097-3110

Chandler JW, Werr W (2017) DORNRÖSCHEN, DORNRÖSCHENLIKE, and PUCHI redundantly control floral meristem identity and organ initiation in Arabidopsis. J Exp Bot 68:3457-3472

Chandler JW, Cole M, Flier A, Grewe B, Werr W (2007) The AP2 transcription factors DORNROSCHEN and DORNROSCHEN-LIKE redundantly control Arabidopsis embryo patterning via interaction with PHAVOLUTA. Development 134:1653-1662

Chandler JW, Comelli P, Jacobs B, Cole M, Werr W (2011) DORNRÖSCHEN-LIKE expression marks Arabidopsis floral organ founder cells and precedes auxin response maxima. Plant Mol Biol 76:171-185

Chen X (2004) A microRNA as a translational repressor of APETALA2 in Arabidopsis flower development. Science 303:2022-2025

Chuck G, Meeley R, Hake S (2008) Floral meristem initiation and meristem cell fate are regulated by the maize $A P 2$ genes $i d s 1$ and sid1. Development 135:3013-3019
Clough SJ, Bent AF (1998) Floral dip: a simplified method for Agrobacterium-mediated transformation of Arabidopsis thaliana. Plant J 16:735-743

Colombo L, Marziani G, Masiero S, Wittich PE, Schmidt RJ, Gorla MS, Pè ME (1998) BRANCHED SILKLESS mediates the transition from spikelet to floral meristem during Zea mays ear development. Plant J 16:355-363

Comelli P, Glowa D, Chandler JW, Werr W (2016) Founder-cell-specific transcription of the DORNRÖSCHEN-LIKE promoter and integration of the auxin response. J Exp Bot 67:143-155

Dereeper A, Guignon V, Blanc G, Audic S, Buffet S, Chevenet F, Dufayard JF, Guindon S, Lefort V, Lescot M et al (2008) Phylogeny.fr: robust phylogenetic analysis for the non-specialist. Nucleic Acids Res 36:W465-W469

Dey S, Corina Vlot A (2015) Ethylene responsive factors in the orchestration of stress responses in monocotyledonous plants. Front Plant Sci 6:640

Drews GN, Bowman JL, Meyerowitz EM (1991) Negative regulation of the Arabidopsis homeotic gene AGAMOUS by the APETALA2 product. Cell 65:991-1002

Elliott RC, Betzner AS, Huttner E, Oakes MP, Tucker WQ, Gerentes D, Perez P, Smyth DR (1996) AINTEGUMENTA, an APETALA2-like gene of Arabidopsis with pleiotropic roles in ovule development and floral organ growth. Plant Cell 8:155-168

Goldshmidt A, Alvarez JP, Bowman JL, Eshed Y (2008) Signals derived from $Y A B B Y$ gene activities in organ primordia regulate growth and partitioning of Arabidopsis shoot apical meristems. Plant Cell 20:1217-1230

Horstman A, Willemsen V, Boutilier K, Heidstra R (2014) AINTEGUMENTA-LIKE proteins: hubs in a plethora of networks. Trends Plant Sci 19:146-157

Irish V (2009) Evolution of petal identity. J Exp Bot 60:2517-2527

Karim MR, Hirota A, Kwiatkowska D, Tasaka M, Aida M (2009) A role for Arabidopsis PUCHI in floral meristem identity and bract suppression. Plant Cell 21:1360-1372

Keck E, McSteen P, Carpenter R, Coen E (2003) Separation of genetic functions controlling organ identity in flowers. EMBO J 22:1058-1066

Kellogg EA (2001) Evolutionary history of the grasses. Plant Physiol 125:1198-1205

Kitazawa MS, Fujimoto K (2014) A developmental basis for stochasticity in floral organ numbers. Front Plant Sci 5:545

Komatsu M, Chujo A, Nagato Y, Shimamoto K, Kyozuka J (2003) FRIZZY PANICLE is required to prevent the formation of axillary meristems and to establish floral meristem identity in rice spikelets. Development 130:3841-3850

Krizek BA (2015) AINTEGUMENTA-LIKE genes have partly overlapping functions with AINTEGUMENTA but make distinct contributions to Arabidopsis thaliana flower development. J Exp Bot 66:4537-4549

Lauter N, Kampani A, Carlson S, Goebel M, Moose SP (2005) microRNA172 down-regulates glossy 15 to promote vegetative phase change in maize. Proc Natl Acad Sci USA 102:9412-9417

Lee DY, An G (2012) Two AP2 family genes, supernumerary bract (SNB) and Osindeterminate spikelet 1 (OsIDS1), synergistically control inflorescence architecture and floral meristem establishment in rice. Plant J 69:445-461

Lee DY, Lee J, Moon S, Park SY, An G (2007) The rice heterochronic gene SUPERNUMERARY BRACT regulates the transition from spikelet meristem to floral meristem. Plant J 49:64-78

Lewis DR, Olex AL, Lundy SR, Turkett WH, Fetrow JS, Muday GK (2013) A kinetic analysis of the auxin transcriptome reveals cell wall remodeling proteins that modulate lateral root development in Arabidopsis. Plant Cell 25:3329-3346

Li Z, Zhang L, Yu Y, Quan R, Zhang Z, Zhang H, Huang R (2011) The ethylene response factor AtERF11 that is transcriptionally 
modulated by the bZIP transcription factor HY5 is a crucial repressor for ethylene biosynthesis in Arabidopsis. Plant $\mathbf{J}$ 68:88-99

Lindsey BE, Rivero L, Calhoun CS, Grotewold E, Brkljacic J (2017) Standardized method for high-throughput sterilization of Arabidopsis seeds. J Vis Exp 128:56587

Litt A (2007) An evaluation of A-function: evidence from the APETALA1 and APETALA2 gene lineages. Int J Plant Sci 168:73-91

Lombardo F, Yoshida H (2015) Interpreting lemma and palea homologies: a point of view from rice floral mutants. Front Plant Sci 6:61

Mantegazza O, Gregis V, Chiara M, Selva C, Leo G, Horner DS, Kater MM (2014) Gene coexpression patterns during early development of the native Arabidopsis reproductive meristem: novel candidate developmental regulators and patterns of functional redundancy. Plant J 79:861-877

Maruyama Y, Yamoto N, Suzuki Y, Chiba Y, Yamazaki K, Sato T, Yamaguchi J (2013) The Arabidopsis transcriptional repressor ERF9 participates in resistance against necrotrophic fungi. Plant Sci 213:79-87

Mathieu J, Yant LJ, Mürdter F, Küttner F, Schmid M (2009) Repression of flowering by the miR172 target SMZ. PLoS Biol 7:e1000148

Matsuhashi S, Sakai S, Kudoh H (2012) Temperature-dependent fluctuation of stamen number in Cardamine hirsuta (Brassicaceae). Int J Plant Sci 173:391-398

McCullough E, Wright KM, Alvarez A, Clark CP, Rickoll WL, Madlung A (2010) Photoperiod-dependent floral reversion in the natural allopolyploid Arabidopsis suecica. New Phytol 186:239-250

McKim SM, Routier-Kierzkowska AL, Monniaux M, Kierzkowski D, Pieper B, Smith RS, Tsiantis M, Hay A (2017) Seasonal regulation of petal number. Plant Physiol 175:886-903

Meng LS, Wang YB, Yao SQ, Liu A (2015) Arabidopsis AINTEGUMENTA mediates salt tolerance by trans-repressing SCABP8. J Cell Sci 128:2919-2927

Müller M, Munné-Bosch S (2015) Ethylene response factors: a key regulatory hub in hormone and stress signaling. Plant Physiol 169:32-41

Nakano T, Suzuki K, Fujimura T, Shinshi H (2006) Genome-wide analysis of the ERF gene family in Arabidopsis and rice. Plant Physiol 140:411-432

Nomura Y, Matsuo N, Banno H (2009) A domain containing the ESR motif in ENHANCER OF SHOOT REGENERATION 1 functions as a transactivation domain. Plant Biotechnol 26:395-401

Ohto MA, Fischer RL, Goldberg RB, Nakamura K, Harada JJ (2005) Control of seed mass by APETALA2. Proc Natl Acad Sci USA 102:3123-3128

Ó’Maoiléidigh DS, Graciet E, Wellmer F (2014) Gene networks controlling Arabidopsis thaliana flower development. New Phytol 201:16-30

Pan Z-J, Chen Y-Y, Du JS, Chen Y-Y, Chung M-C, Tsai W-C, Wang C-N, Chen H-H (2014) Flower development of Phalaenopsis orchid involves functionally divergent SEPALLATA-like genes. New Phytol 202:1024-1042

Park SJ, Eshed Y, Lippman ZB (2014) Meristem maturation and inflorescence architecture - lessons from the Solanaceae. Curr Opin Plant Biol 17:70-77

Peaucelle A, Morin H, Traas J, Laufs P (2007) Plants expressing a miR164-resistant $C U C 2$ gene reveal the importance of post-meristematic maintenance of phyllotaxy in Arabidopsis. Development 134: $1045-1050$

Pieper B, Monniaux M, Hay A (2016) The genetic architecture of petal number in Cardamine hirsuta. New Phytol 209:395-406

Plackett ARG, Powers SJ, Phillips AL, Wilson ZA, Hedden P, Thomas SG (2018) The early inflorescence of Arabidopsis thaliana demonstrates positional effects in floral organ growth and meristem patterning. Plant Reprod 31:171-191
Pouteau S, Albertini C (2011) An assessment of morphogenetic fluctuation during reproductive phase change in Arabidopsis. Ann Bot 107:1017-1027

Pouteau S, Tooke F, Battey N (1998) Quantitative control of inflorescence formation in Impatiens balsamina. Plant Physiol 118:1191-1201

Reinhardt D, Mandel T, Kuhlemeier C (2000) Auxin regulates the initiation and radial position of plant lateral organs. Plant Cell 12:507-518

Ren D, Li Y, Zhao F, Sang X, Shi J, Wang N, Guo S, Ling Y, Zhang C, Yang Z, He G (2013) MULTI-FLORET SPIKELET1, which encodes an AP2/ERF protein, determines spikelet meristem fate and sterile lemma identity in rice. Plant Physiol 162:872-884

Sauquet H, von Balthazar M, Magallón S, Doyle JA, Endress PK, Bailes EJ, Barroso de Morais E, Bull-Hereñu K, Carrive L, Chartier M et al (2017) The ancestral flower of angiosperms and its early diversification. Nat Commun 8:16047

Schultz EA, Haughn GW (1991) $L E A F Y$, a homeotic gene that regulates inflorescence development in Arabidopsis. Plant Cell 3:771-781

Shrestha R, Gómez-Ariza J, Brambilla V, Fornara F (2014) Molecular control of seasonal flowering in rice, Arabidopsis and temperate cereals. Ann Bot 114:1445-1458

Smyth DR, Bowman JL, Meyerowitz EM (1990) Early flower development in Arabidopsis. Plant Cell 2:755-767

Teotia S, Tang G (2015) To bloom or not to bloom: role of microRNAs in plant flowering. Mol Plant 8:359-377

Tooke F, Ordidge M, Chiurugwi T, Battey N (2005) Mechanisms and function of flower and inflorescence reversion. J Exp Bot $56: 2587-2599$

Überlacker B, Werr W (1996) Vectors with rare-cutter restriction enzyme sites for expression of open reading frames in transgenic plants. Mol Breed 2:293-295

Wellmer F, Alves-Ferreira M, Dubois A, Riechmann JL, Meyerowitz EM (2006) Genome-wide analysis of gene expression during early Arabidopsis flower development. PLoS Genet 2:e117

Whipple CJ (2017) Grass inflorescence architecture and evolution: the origin of novel signaling centers. New Phytol 216:367-372

Whipple CJ, Zanis MJ, Kellogg EA, Schmidt RJ (2007) Conservation of B class gene expression in the second whorl of a basal grass and outgroups links the origin of lodicules and petals. Proc Natl Acad Sci USA 104:1081-1086

Wong CE, Bhalla PL, Ottenhof H, Singh MB (2008) Transcriptional profiling of the pea shoot apical meristem reveals processes underlying its function and maintenance. BMC Plant Biol 8:73

Yadav RK, Girke T, Pasala S, Xie M, Reddy GV (2009) Gene expression map of the Arabidopsis shoot apical meristem cell niche. Proc Natl Acad Sci USA 106:4941-4946

Yant L, Mathieu J, Dinh TT, Ott F, Lanz C, Wollmann H, Chen X, Schmid M (2010) Orchestration of the floral transition and floral development in Arabidopsis by the bifunctional transcription factor APETALA2. Plant Cell 22:2156-2170

Zhang JY, Pan D-L, Wang G, Xuan J-P, Wang T, Guo Z-R (2017) Genome-wide analysis and expression pattern of the AP2/ERF gene family in kiwifruit under waterlogging stress treatment. Int J Environ Agric Res 3:83-90

Zhou X, Zhang ZL, Park J, Tyler L, Yusuke J, Qiu K, Nam EA, Lumba S, Desveaux D, McCourt P et al (2016) The ERF11 transcription factor promotes internode elongation by activating gibberellin biosynthesis and signaling. Plant Physiol 171:2760-2770

Publisher's Note Springer Nature remains neutral with regard to jurisdictional claims in published maps and institutional affiliations. 